\title{
Closed-form Solutions of the Time-fractional Standard Black-Scholes Model for Option Pricing using He-separation of Variable Approach
}

\author{
S. O. EDEKI ${ }^{1 *}$, G. O. AKINLABI ${ }^{1}$, F. O. EGARA ${ }^{2}$, A. C. NZEADIBE ${ }^{2}$ \\ ${ }^{1}$ Department of Mathematics, Covenant University, Ota, NIGERIA \\ ${ }^{2}$ Department of Science Education, University of Nigeria, Nsukka, NIGERIA
}

\begin{abstract}
The Black-Scholes option pricing model in classical form remains a benchmark model in Financial Engineering and Mathematics concerning option valuation. Though, it has received a series of modifications as regards its initial constancy assumptions. Most of the resulting modifications are nonlinear or time-fractional, whose exact or analytical solutions are difficult to obtain. This paper, therefore, presents exact (closed-form) solutions to the time-fractional classical Black-Scholes option pricing model by means of the He-Separation of Variable Transformation Method (HSVTM). The HSVTM combines the features of the He's polynomials, the Homo-separation variable, the modified DTM, which increases the efficiency and effectiveness of the proposed method. The proposed method is direct and straight forward. Hence, it is recommended for obtaining solutions to financial models resulting from either Ito or Stratonovich Stochastic Differential Equations (SDEs).
\end{abstract}

Key-Words: - Option pricing, Black-Scholes model, exact solutions, fractional calculus Received: August 16, 2019. Revised: February 3, 2020. Accepted: February 17, 2020. Published: February 28, 2020.

\section{Introduction}

Almost all aspects of applied mathematics have witnessed the emergence of Fractional Calculus (FC) as an important generalization of the classical calculus [1-4]. The derivative order(s) associated with FC go beyond the set of natural numbers, the point estimate, and so on. Instead, the orders can be defined in real and complex spaces, while an interval estimate is considered [5-8]. This creates rooms for memory settings of the systems. The applications of FC are widely seen in [9-11]. In financial mathematics, Jumaris [12,13] introduced FC to option pricing with its base in the BlackScholes pricing model (Financial derivatives). The Black-Scholes Model (BSM) for European option pricing and valuation plays a notable role in risk and portfolio management [14-17]. Though, some of the BSM underlying assumptions when relaxed leads to more complex or nonlinear versions. Hence, the need for effective and efficient numerical, semiapproximate methods of solution. In literature, a lot of solution methods have been considered by a good number of researchers. These include: Adomian decomposition method (ADM), variational iteration method (VIM), Modified ADM (MADM), homotopy perturbation method (HPM), Differential transformation method (DTM), projected DTM (PDTM) [18-25]. He's polynomials method was initiated in $[26,27]$ by Ghorbani et al., where the nonlinear terms were expressed as series of polynomials calculated with the aid of HPM. The He's polynomials are noted to be compatible with the so-called Adomian's polynomials. Though, they are easier to be computed and are user friendly to a greater extent.

Other numerical approaches with more extensive applications to modelling situations in sciences, engineering, finance, and environmental management are considered in terms of analytical or approximate solutions [28-37].

\section{The Classical Black-Scholes Model}

The solution of the Black-Scholes model is used for describing the value of option mainly of European type [38]. The solution solves the model of the form:

$$
\frac{\partial f}{\partial \tau}+\frac{1}{2} S^{2} \sigma^{2} \frac{\partial^{2} f}{\partial S^{2}}+r S \frac{\partial f}{\partial S}-r f=0
$$

with the following as defined: $f=f(S, \tau)$ represents the value of the underlying $S$, at a particular time, $\tau$ such that $\tau \in[0, t], f \in C^{2,1}[R \times[0, T]],(S, \tau) \in R^{+} \times(0, T)$ for a payoff function $p_{f}(S, t)$, and expiration price, $E$ such that: 
$p_{f}(S, t)=\left\{\begin{array}{l}(E-S)^{+}, \text {for European put option, } \\ (S-E)^{+}, \text {for European call option. }\end{array}\right.$

In (2), $\left(S_{*}\right)^{+}$represents the maximum between

$S_{*}$ and 0 in terms of values, for the underlying asset $S=S(t)$, the volatility is $\sigma, r$ is taken as the risk-free interest rate, meanwhile, the maturity time is $T$.

In this work, we will look at a generalization of (1) regarding fractional order in terms of real and complex order of the derivatives. This will be regarded as a non-integer (time-fractional) BlackScholes model (TFBSM) following the form:

$\frac{\partial^{\alpha} \Psi}{\partial \tau^{\alpha}}+m_{1}(S, \delta) \frac{\partial^{2} \Psi}{\partial S^{2}}+m_{2}(S, r) \frac{\partial \Psi}{\partial S}=r \Psi, \alpha>0$

subject to an attributed initial or boundary conditions, $m_{i}(\cdot, \cdot), i=0,1,2,3, \cdots$, are non-zero functions.

Recently, Ghandehari and Ranjbar [39] presented the exact solution of the option pricing model built on the Fractional Black-Scholes (FBS) equation employing a modified Homotopy Perturbation Method (HPM). In their method, they obtained the exact solutions basically with the aid of green function by combining the separation of variables method with HPM [39].

Ouafoudi and Gao [40] introduced two solution methods viz: modified HPM and Homotopy Perturbation combined with Sumudu transform for handling the same option pricing model as considered in [39]. Both views of [39] and [40] required the application of green function. The new approach in this present work aims at providing exact solutions of the time-fractional classical Black-Scholes option pricing model by means of He-Separation of Variable Transformation Method (HSVTM). The HSVTM combines the basic features of the He's polynomials, the Homoseparation variable, and the modified Differential Transform Method without the concept and application of green function. Here, the fractional derivative is defined in the sense of Caputo.

\section{Remarks on the He's Polynomial Solution Method}

Suppose a general form is considered as follows:

$$
\Lambda(\psi)=0
$$

for a differential or an integral operator, $\Lambda$ and $H(\psi, p)$ denotes a convex homotopy given as:

$$
H(\psi, p)=p \Lambda(\psi)+(1-p) \Omega(\psi)
$$

where $\Omega(\psi)$ is a known operator (functional) with $\psi_{0}$ as a solution. Therefore, we get:

$$
\left.\begin{array}{l}
H(\psi, 0)=\Omega(\psi) \\
H(\psi, 1)=\Lambda(\psi)
\end{array}\right\}
$$

whenever $H(\psi, p)=0$ is satisfied, and the parameter $p \in(0,1]$ is embedded. According to HPM in [26, 27], the parameter, $p$ is used in the expansion of:

$\psi=\sum_{j=0}^{\infty} p^{j} \psi_{j}=\psi_{0}+p \psi_{1}+p^{2} \psi_{2}+\cdots$

From (7) we have the solution as $p \rightarrow 1$. Though, the convergence of (7) as $p \rightarrow 1$ has already been considered in [24].

The method considers $N(\psi)$ as the nonlinear term given as:

$N(\psi)=\sum_{j=0}^{\infty} p^{j} H_{j}=H_{o}+p^{1} H_{1}+p^{2} H_{2}+\cdots$

where the He's polynomials, $H_{k}{ }^{\prime} s$ can be obtained using:

$$
H_{k}\left(\psi_{0}, \psi_{1}, \cdots, \psi_{k}\right)=\frac{1}{k !} \frac{\partial^{k}}{\partial p^{k}}\left(N\left(\sum_{j=0}^{k} p^{j} \psi_{j}\right)\right)_{p=0}, .
$$

\subsection{The method on FDE}

Consider the general form of the time-fractional differential equation (GTFDE) of the form:

$$
\left\{\begin{array}{l}
D_{t}^{\alpha} u(x, t)=L u(x, t)+N u(x, t)+f(x, t), \\
u(x, 0)=g(x),
\end{array}\right.
$$

where $D_{t}^{\alpha}$ is the fractional differential operator of order $\alpha \in(0,1]$ in the sense of Caputo, $L$ is a linear operator, $N$ is a nonlinear operator, $f(x, t)$ is a source term and $u(x, t)$ is a supposed function satisfying (3.7).

Suppose $H(\psi, p) ; \psi(x, t)$ is defined as a convex homotopy such that:

where,

$$
H(\psi, p)=p \Lambda(\psi)+(1-p) \Omega(\psi)
$$




$$
\Lambda(\psi)=D_{t}^{\alpha} u(x, t)-L u(x, t)-N u(x, t)-f(x, t)
$$

and $\Lambda(\psi)$ is a functional operator with $\psi_{0}$ as known solution such that:

$$
\Omega(\psi)=D_{t}^{\alpha} u(x, t)-D_{t}^{\alpha} u(x, 0) .
$$

We remarked that:

$$
H(\psi, p)=\left\{\begin{array}{l}
\Omega(\psi), \text { for } p=0, \\
\Lambda(\psi), \text { for } p=1
\end{array}\right.
$$

For $H(\psi, p) \equiv 0$, and using (12) and (13), we have:

$$
\begin{aligned}
& p \Lambda(\psi)+(1-p) \Omega(\psi)=0 \\
& \Rightarrow \quad p\left(D_{t}^{\alpha} u(x, t)-L u(x, t)-N u(x, t)-f(x, t)\right) \\
& \quad+(1-p)\left(D_{t}^{\alpha} u(x, t)-D_{t}^{\alpha} u(x, 0)\right)=0
\end{aligned} .
$$

Expanding and simplifying (15) give:

$$
\begin{aligned}
& D_{t}^{\alpha} u(x, t)=D_{t}^{\alpha} u(x, 0) \\
& \quad+p\left(L u(x, t)+N u(x, t)+f(x, t)-D_{t}^{\alpha} u(x, 0)\right)
\end{aligned}
$$

where $u(x, 0)$ is an initial approximation of $(10)$. In an integral form, (16) is expressed as:

$$
u(x, t)=u(x, 0)+p J_{t}^{\alpha}\left(\begin{array}{l}
L u(x, t)+N u(x, t) \\
+f(x, t)-D_{t}^{\alpha} u(x, 0)
\end{array}\right)
$$

where the nonlinear term is as defined above.

Applying convex homotopy method to (17) with $p \in(0,1]$ as an embedded parameter yields:

$$
\begin{aligned}
\sum_{n=0}^{\infty} p^{n} u_{n}(x, t)=u(x, 0) \\
+p^{n+1} J_{t}^{\alpha}\left(\begin{array}{l}
L\left(\sum_{n=0}^{\infty} u_{n}(x, t)\right) \\
+N\left(\sum_{n=0}^{\infty} u_{n}(x, t)\right)+\phi(x)
\end{array}\right)
\end{aligned}
$$

where $\phi(x)=f(x, t)-D_{t}^{\alpha} u(x, 0)$.

\subsection{Procedures for the Exact Solution}

From (18), $p^{0}: u_{0}=u(x, 0)$. It is obvious that $u_{0}=u(x, 0)$ is the initial approximation (condition) of (10). To reduce the FDE (10) to ODE of the equivalent form, the exact solution is therefore defined as:

$$
u_{*}(x, t)=u(x, 0) \lambda_{1}(t)+u^{\prime}(x, 0) \lambda_{2}(t) \text {. }
$$

The functions $\lambda_{1}(t)$ and $\lambda_{2}(t)$ are to be determined. Thus, $u_{*}(x, t)$ satisfies (3.7). Hence,

$D_{t}^{\alpha} u_{*}(x, t)=L u_{*}(x, t)+N u_{*}(x, t)+f(x, t)$.

That implies that:

$$
\begin{aligned}
D_{t}^{\alpha}\left(\begin{array}{l}
u(x, 0) \lambda_{1}(t) \\
+u^{\prime}(x, 0) \lambda_{2}(t)
\end{array}\right) & =L\left(\begin{array}{l}
u(x, 0) \lambda_{1}(t) \\
+u^{\prime}(x, 0) \lambda_{2}(t)
\end{array}\right) \\
& +N\left(\begin{array}{l}
u(x, 0) \lambda_{1}(t) \\
+u^{\prime}(x, 0) \lambda_{2}(t)
\end{array}\right)+f(x, t) .
\end{aligned}
$$

Since the assumed solution:

$u(x, t)=\sum_{\substack{i=0 \\ p_{i} \rightarrow 1}}^{\infty} u_{i} p_{i}$

satisfies the initial condition, we have:

$u_{*}(x, 0)=u(x, 0) \lambda_{1}(0)+u^{\prime}(x, 0) \lambda_{2}(0)$.

Thus,

$\left\{\begin{array}{l}\lambda_{1}(0)=1 \\ \lambda_{2}(0)=0 .\end{array}\right.$

Though, we have from (16) that:

$D_{t}^{\alpha} u_{1}(x, t)=D_{t}^{\alpha} u_{0}(x, 0)$

$$
-\left(L u_{0}(x, t)+N u_{0}(x, t)+f(x, t)\right) \equiv 0 \cdot
$$

Hence, simplifying (21) by putting (22) in (24) gives:

$$
\begin{aligned}
&\left\{\begin{array}{l}
u(x, 0) D_{t}^{\alpha}\left(\lambda_{1}(t)\right) \\
+u^{\prime}(x, 0) D_{t}^{\alpha}\left(\lambda_{2}(t)\right)
\end{array}\right\}=L\left(\begin{array}{l}
u(x, 0) \lambda_{1}(t) \\
+u^{\prime}(x, 0) \lambda_{2}(t)
\end{array}\right) \\
&+N\left(\begin{array}{l}
u(x, 0) \lambda_{1}(t) \\
+u^{\prime}(x, 0) \lambda_{2}(t)
\end{array}\right)+f(x, t) .
\end{aligned}
$$

Equation (25) is thus, the fractional ODE (FODE) resulting from the fractional PDE in (10). Solving (25) may either lead to an IVP (ODE) or a system of ODEs. We will resort to Projected Differential Transform Method (PDTM) [25, 38, 41] for ease of computation as regards (25). Hence, suppose is an analytic function at in a given domain say, $D$, then, the projected DTM of $\psi(x, t)$ with respect to $t$ at $t_{*}$ is defined and denoted by:

$$
\Xi(x, j)=\frac{1}{j !}\left[\frac{\partial^{j} \psi(x, t)}{\partial t^{j}}\right]_{t=t_{0}}
$$

such that:

$$
\psi(x, j)=\sum_{j=0}^{\infty} \Xi(x, j)\left(t-t_{0}\right)^{j}
$$


where (27) is known as the projected differential inverse transform of $\Xi(x, j)$ with respect to the time parameter $t$.

\section{Applications}

In this section, the following time-fractional BlackScholes equations are considered.

Problem 4.1: A linear Black-Scholes equation of the following form is considered:

$$
\frac{\partial^{\alpha} w}{\partial t^{\alpha}}+x^{2} \frac{\partial^{2} w}{\partial x^{2}}+\frac{1}{2} x \frac{\partial w}{\partial x}-w=0
$$

subject to:

$$
w(x, 0)=\max \left(x^{3}, 0\right)=\left\{\begin{array}{l}
x^{3}, \text { for } x>0 \\
0, \text { for } x \leq 0
\end{array} .\right.
$$

\section{Procedure w.r.t Problem 4.1:}

Choose $w_{*}(x, t)$ as an initial approximation to $(28)$ such that:

$$
w_{*}(x, t)=\left\{\begin{array}{l}
\max \left(x^{3}, 0\right) \lambda_{1}(t) \\
+3 \max \left(x^{2}, 0\right) \lambda_{2}(t)
\end{array}\right\} .
$$

Hence, (28) becomes:

$$
\begin{gathered}
0=\left\{\begin{array}{l}
\frac{\partial^{\alpha}}{\partial t^{\alpha}}\left(\max \left(x^{3}, 0\right) \lambda_{1}(t)+3 \max \left(x^{2}, 0\right) \lambda_{2}(t)\right) \\
+x^{2} \frac{\partial^{2}}{\partial x^{2}}\left(\max \left(x^{3}, 0\right) \lambda_{1}(t)+3 \max \left(x^{2}, 0\right) \lambda_{2}(t)\right) \\
+\frac{1}{2} x \frac{\partial}{\partial x}\left(\max \left(x^{3}, 0\right) \lambda_{1}(t)+3 \max \left(x^{2}, 0\right) \lambda_{2}(t)\right) \\
-\left(\max \left(x^{3}, 0\right) \lambda_{1}(t)+3 \max \left(x^{2}, 0\right) \lambda_{2}(t)\right)
\end{array}\right\} \\
=\left\{\begin{array}{l}
\frac{\partial^{\alpha}}{\partial t^{\alpha}}\left(\max \left(x^{3}, 0\right) \lambda_{1}(t)+3 \max \left(x^{2}, 0\right) \lambda_{2}(t)\right) \\
+x^{2} \frac{\partial^{2}}{\partial x^{2}}\left(\max \left(x^{3}, 0\right) \lambda_{1}(t)+3 \max \left(x^{2}, 0\right) \lambda_{2}(t)\right) \\
+\frac{1}{2} x \frac{\partial}{\partial x}\left(\max \left(x^{3}, 0\right) \lambda_{1}(t)+3 \max \left(x^{2}, 0\right) \lambda_{2}(t)\right) \\
-\left(\max \left(x^{3}, 0\right) \lambda_{1}(t)+3 \max \left(x^{2}, 0\right) \lambda_{2}(t)\right)
\end{array}\right\} .
\end{gathered}
$$

$$
\begin{aligned}
\Rightarrow & \\
0 & \left(x^{3} \frac{d^{\alpha}}{d t^{\alpha}} \lambda_{1}(t)+3 x^{2} \frac{d^{\alpha}}{d t^{\alpha}} \lambda_{2}(t)\right) \\
& +\left(6 x^{3} \lambda_{1}(t)+6 x^{2} \lambda_{2}(t)\right) \\
& +\frac{3 x^{3}}{2} \lambda_{1}(t)+3 x^{2} \lambda_{2}(t)-x^{3} \lambda_{1}(t)-3 x^{2} \lambda_{2}(t) \\
= & \left(x^{3} \frac{d^{\alpha}}{d t^{\alpha}} \lambda_{1}(t)+3 x^{2} \frac{d^{\alpha}}{d t^{\alpha}} \lambda_{2}(t)\right) \\
& +6 x^{2} \lambda_{2}(t)+\frac{13 x^{3}}{2} \lambda_{1}(t) .
\end{aligned}
$$

Thus,

$$
\begin{aligned}
0 \equiv & x^{3}\left(\frac{d^{\alpha}}{d t^{\alpha}} \lambda_{1}(t)+\frac{13}{2} \lambda_{1}(t)\right) \\
& +3 x^{2}\left(\frac{d^{\alpha}}{d t^{\alpha}} \lambda_{2}(t)+2 \lambda_{2}(t)\right)
\end{aligned}
$$

We therefore obtain the FODE system:

$$
\begin{aligned}
& \left\{\begin{array}{l}
\frac{d^{\alpha}}{d t^{\alpha}} \lambda_{1}(t)+\frac{13}{2} \lambda_{1}(t)=0, \\
\lambda_{1}(0)=1
\end{array}\right. \\
& \left\{\begin{array}{l}
\left.\frac{d^{\alpha}}{d t^{\alpha}} \lambda_{2}(t)+2 \lambda_{2}(t)\right)=0 \\
\lambda_{2}(0)=0
\end{array} .\right.
\end{aligned}
$$

From (34), it is obvious that $\lambda_{2}(t)=0$. But solving (33) using the transformation properties [38] with $\Xi_{1}(h)$ as the differential transform of $\lambda_{1}(t)$ gives:

$\Xi_{1}(1+h)=\frac{\Gamma(1+\alpha h)}{\Gamma(1+\alpha(1+h))}\left(-6.5 \Xi_{1}(h)\right)$.

Thus,

$$
\begin{aligned}
\Xi_{1}(p)= & \frac{(-6.5)^{p}}{\Gamma(1+p \alpha)}, p \geq 1 . \\
\Rightarrow \lambda_{1}(t) & =\sum_{p=0}^{\infty} \Xi_{1}(p) t^{\alpha p} \\
& =\sum_{p=0}^{\infty} \frac{(-6.5)^{p}}{\Gamma(1+p \alpha)} t^{\alpha p} \\
& =\sum_{p=0}^{\infty} \frac{\left(-6.5 t^{\alpha}\right)^{p}}{\Gamma(1+p \alpha)} \\
& =E_{\alpha}\left(-6.5 t^{\alpha}\right) .
\end{aligned}
$$

So, using (34) and (37) in (30) gives: 


$$
\begin{aligned}
w_{*}(x, t) & =\max \left(x^{3}, 0\right) \sum_{p=0}^{\infty} \frac{\left(-6.5 t^{\alpha}\right)^{p}}{\Gamma(1+p \alpha)} \\
& =\max \left(x^{3}, 0\right) E_{\alpha}\left(-6.5 t^{\alpha}\right) \\
& =x^{3} E_{\alpha}\left(-6.5 t^{\alpha}\right)
\end{aligned}
$$

where $E_{\alpha}\left(-k t^{\alpha}\right)$ denotes a one parameter MittagLeffler function.

\section{Problem 4.2:}

A linear Black-Scholes equation model the following form is considered:

$$
\frac{\partial^{\alpha} w}{\partial t^{\alpha}}=\frac{\partial^{2} w}{\partial x^{2}}+(k-1) \frac{\partial w}{\partial x}-k w
$$

subject to:

$$
w(x, 0)=\max \left(e^{x}-1,0\right) .
$$

\section{Procedure w.r.t Problem 4.2:}

Choose $w_{*}(x, t)$ as an initial approximation to (39) such that:

$$
w_{*}(x, t)=\left\{\begin{array}{l}
\max \left(0, e^{x}-1\right) \lambda_{1}(t) \\
+\max \left(e^{x}, 0\right) \lambda_{2}(t)
\end{array}\right\} .
$$

Hence, (39) becomes:

$$
\begin{aligned}
& 0=\left\{\begin{array}{l}
\frac{\partial^{\alpha}}{\partial t^{\alpha}}\left(\max \left(0, e^{x}-1\right) \lambda_{1}(t)+\max \left(e^{x}, 0\right) \lambda_{2}(t)\right) \\
-\frac{\partial^{2}}{\partial x^{2}}\left(\max \left(0, e^{x}-1\right) \lambda_{1}(t)+\max \left(e^{x}, 0\right) \lambda_{2}(t)\right) \\
+(1-k) \frac{\partial w}{\partial x}\left(\begin{array}{l}
\max \left(0, e^{x}-1\right) \lambda_{1}(t) \\
+\max \left(e^{x}, 0\right) \lambda_{2}(t)
\end{array}\right) \\
+k\left(\max \left(0, e^{x}-1\right) \lambda_{1}(t)+\max \left(e^{x}, 0\right) \lambda_{2}(t)\right) .
\end{array}\right\} \\
& \Rightarrow \\
& 0=\max \left(0, e^{x}-1\right) \frac{d^{\alpha}}{\partial t^{\alpha}} \lambda_{1}(t)+\max \left(e^{x}, 0\right) \frac{d^{\alpha}}{\partial t^{\alpha}} \lambda_{2}(t) \\
& -k \max \left(e^{x}, 0\right) \lambda_{1}(t)+k \max \left(0, e^{x}-1\right) \lambda_{1}(t) .
\end{aligned}
$$

Thus,

$$
0=\left\{\begin{array}{l}
\max \left(e^{x}-1,0\right)\left(\frac{d^{\alpha}}{\partial t^{\alpha}} \lambda_{1}(t)+k \lambda_{1}(t)\right) \\
+\max \left(e^{x}, 0\right)\left(\frac{d^{\alpha}}{\partial t^{\alpha}} \lambda_{2}(t)-k \lambda_{1}(t)\right)
\end{array}\right\} .
$$

$$
\left\{\begin{array}{l}
\frac{d^{\alpha}}{d t^{\alpha}} \lambda_{1}(t)+k \lambda_{1}(t)=0, \\
\lambda_{1}(0)=1
\end{array}\right.
$$

and

$$
\left\{\begin{array}{l}
\frac{d^{\alpha}}{d t^{\alpha}} \lambda_{2}(t)-k \lambda_{1}(t)=0, \\
\lambda_{2}(0)=0 .
\end{array}\right.
$$

From (45), the relation:

$$
\begin{aligned}
& \left\{\begin{array}{l}
\Xi_{1}(1+h)=\frac{\Gamma(1+\alpha h)}{\Gamma(1+\alpha(1+h))}\left(-k \Xi_{1}(h)\right), \\
\Xi_{1}(0)=1 .
\end{array}\right. \\
& \Rightarrow \quad \Xi_{1}(p)=\frac{(-k)^{p}}{\Gamma(1+p \alpha)}, p \geq 0 .
\end{aligned}
$$

Therefore,

$$
\left.\begin{array}{rl}
\lambda_{1}(t) & =\sum_{p=0}^{\infty} \Xi_{1}(p) t^{\alpha p} \\
& =\sum_{p=0}^{\infty} \frac{\left(-k t^{\alpha}\right)^{p}}{\Gamma(1+p \alpha)}=E_{\alpha}\left(-k t^{\alpha}\right)
\end{array}\right\}
$$

Similarly, from (46), the relation:

$$
\left.\begin{array}{l}
\left\{\begin{array}{l}
\Xi_{2}(1+h)=\frac{\Gamma(1+\alpha h)}{\Gamma(1+\alpha(1+h))}\left(k \Xi_{1}(h)\right), \\
\Xi_{1}(0)=1, \Xi_{2}(0)=0 .
\end{array}\right. \\
\Rightarrow \quad \Xi_{2}(p)=\frac{-(-k)^{p}}{\Gamma(1+p \alpha)}, p \geq 1 . \\
\quad \lambda_{2}(t)=\sum_{p=0}^{\infty} \Xi_{2}(p) t^{\alpha p} \\
\quad=-\sum_{p=1}^{\infty} \frac{\left(-k t^{\alpha}\right)^{p}}{\Gamma(1+p \alpha)}
\end{array}\right\}
$$

But

$$
\begin{aligned}
& \sum_{p=0}^{\infty} \frac{\left(-k t^{\alpha}\right)^{p}}{\Gamma(1+p \alpha)}=1+\sum_{p=1}^{\infty} \frac{\left(-k t^{\alpha}\right)^{p}}{\Gamma(1+p \alpha)} . \\
\therefore \quad & 1-\sum_{p=0}^{\infty} \frac{\left(-k t^{\alpha}\right)^{p}}{\Gamma(1+p \alpha)}=-\sum_{p=1}^{\infty} \frac{\left(-k t^{\alpha}\right)^{p}}{\Gamma(1+p \alpha)} .
\end{aligned}
$$

So, putting (54) in (52) gives:

$$
\left.\begin{array}{rl}
\lambda_{2}(t) & =1-\sum_{p=0}^{\infty} \frac{\left(-k t^{\alpha}\right)^{p}}{\Gamma(1+p \alpha)} \\
& =1-E_{\alpha}\left(-k t^{\alpha}\right) .
\end{array}\right\}
$$

We therefore obtain the FODE system: 
Hence,

$$
W_{*}(x, t)=\left\{\begin{array}{l}
\max \left(0, e^{x}-1\right) E_{\alpha}\left(-k t^{\alpha}\right) \\
+\max \left(e^{x}, 0\right)\left(1-E_{\alpha}\left(-k t^{\alpha}\right)\right)
\end{array}\right\} .
$$

Here, the presentations of the graphical views of the solutions $w=w(x, t)$ are given (see figures 1-4).

Fig.1 and Fig.2 are for problem 4.1, while Fig.3 and Fig.4 are for problem 4.2. For each considered case, the same interval is applied for $x$ but different intervals are used for $t$.

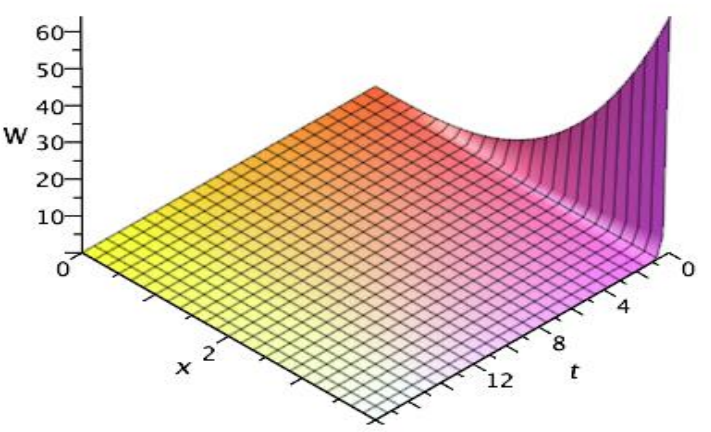

Fig. 1: $w: o \leq t \leq 18, o \leq x \leq 4$

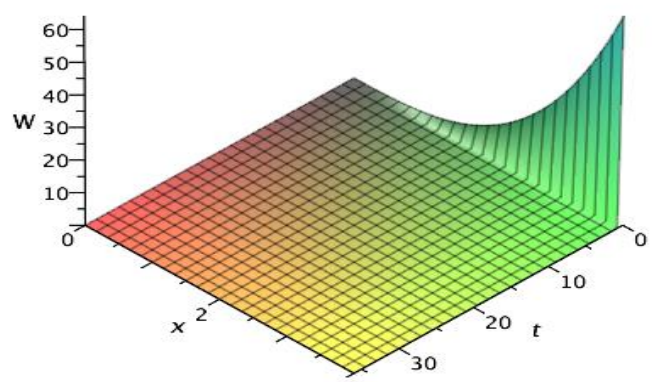

Fig. 2: $w: o \leq t \leq 36, o \leq x \leq 4$

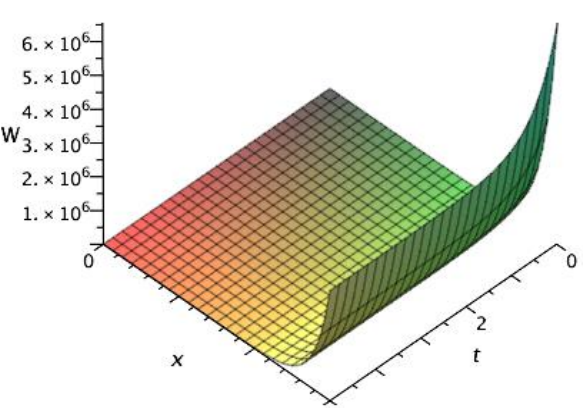

Fig. 3: $w: o \leq t \leq 5, o \leq x \leq 15$

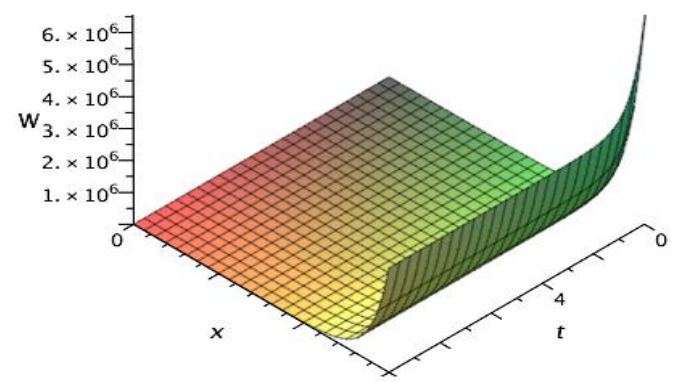

Fig. 4: $w: o \leq t \leq 10, o \leq x \leq 15$

\section{Concluding Remarks}

In this paper, a new exact solution method is proposed. The method presented the exact solutions of the time-fractional classical Black-Scholes option pricing model by means of the He-Separation of Variable Transformation Method (HSVTM). This is an extension of the conference paper-approach in [42]. The HSVTM combined the basic properties of the He's polynomials, the Homo-separation variable, and the modified DTM. The engendered fractional derivative is defined in the sense of Caputo, which increases the efficiency and effectiveness of the proposed method. The merits of the HSVTM are numerous. These include direct and straightforwardness in its application. Besides, no knowledge of green function, linearization, or Lagrange multiplier is required. Hence, it is recommended for obtaining solutions of financial models resulting from either Ito or Stratonovich Stochastic Differential Equations (SDEs). 


\section{Acknowledgements}

With sincerity, the authors wish to thank Covenant University for all forms of support. The authors also wish to thank the anonymous referee(s)/reviewer(s) for constructive remarks.

\section{References:}

[1] S. Kumar, A. Yildirim, Y. Khan, H. Jafari, K. Sayevand, and L. Wey, Analytical solution of fractional Black-Scholes, European option pricing equation by using Laplace transform, $J$. of Fractional Calculus and Applications, 2, (2012), 1-9.

[2] S.O. Edeki, O.O. Ugbebor, and E.A. Owoloko, Analytical Solution of the Time-fractional Order Black-Scholes Model for Stock Option Valuation on No Dividend Yield Basis, IAENG International Journal of Applied Mathematics, 47 (4), (2017), 407-416.

[3] I. Podlubny. Fractional Differential Equation, Academic Press, San Diego (1999).

[4] R.M. Jena and S. Chakraverty. Residual Power Series Method for Solving Time-fractional Model of Vibration Equation of Large Membranes, J. Appl. Comput. Mech. 5 (4), (2019), 603-615.

[5] D. Baleanu, K. Diethelm, E. Scalas, J.J. Trujillo, Fractional Calculus: Models and Numerical Methods, World Scientific Publishing Company: Boston, MA, USA, (2012).

[6] B. K. Singh, and V.K. Srivastava, Approximate series solution of multi-dimensional, time fractional-order (heat-like) diffusion equations using FDRM. Royal Society Open Science, 2, (2015), 140-511.

[7] E. Ozbilge, A. Demir, An inverse source problem in time-space fractional differential equations, Applied Mathematics and Information Sciences, 12 (3), (2018), 587-591.

[8] S.O. Edeki, and I. Adinya, Coupled transform for approximate-analytical solutions of a timefractional one-factor markovian bond pricing model, WSEAS Transactions on Systems and Control, 14, (2019), 202-208.

[9] R.M. Jena and S. Chakraverty, D. Baleanu, On New Solutions of Time-Fractional Wave Equations Arising in Shallow Water Wave Propagation, Mathematics, 7, (2019), 722.

[10] R.M. Jena, and S. Chakraverty. A new iterative method based solution for fractional BlackScholes option pricing equations (BSOPE), $S N$ Applied Sciences, 1, (2019), 95.
[11] S. Javeed, D. Baleanu, A. Waheed, M.S. Khan, H. Affan. Analysis of Homotopy Perturbation Method for Solving Fractional Order Differential Equations, Mathematics, 7, 40, (2019).

[12] G. Jumarie, Fractional partial differential equations and modified Riemann-Liouville derivative new methods for solution, Journal of Applied Mathematics and Computing, 24 (1-2), (2007), 31-48.

[13] G. Jumarie, Modified Riemann-Liouville Derivative and Fractional Taylor series of Nondifferentiable Functions Further Results, Computers and Mathematics with Applications, 51 (9-10), (2006), 1367-1376.

[14] J.C. Hull, Options, Futures, and Other Derivatives, New Jersey: Prentice-Hall, 1999.

[15] F. Black, M. Scholes, The pricing of options and corporate liabilities, Journal of Political Economy, 81, (1973), 637-654.

[16] R.C. Merton, Theory of rational options pricing, The Bell Journal of Economics and Management Science, 4 (1), (1973), 141-183.

[17] S.O. Edeki, E.A. Owoloko, and O.O. Ugbebor, The Modified Black-Scholes Model via Constant Elasticity of Variance for Stock Options Valuation, AIP Conference Proceedings, 1705, (2016), Article number 4940289.

[18] O. González-Gaxiola, and J. R. de Chávez J. A. Santiago, A Nonlinear Option Pricing Model Through the Adomian Decomposition Method, International Journal of Applied and Computational Mathematics, DOI 10.1007/s40819-015-0070-6: (2015).

[19] V. Gülkaç, The homotopy perturbation method for the Black-Scholes equation, Journal of Statistical Computation and Simulation 80 (12), ( 2010), 1349-1354.

[20] M. R. Rodrigo, R. S. Mamon, An alternative approach to solving the Black-Scholes equation with time-varying parameters, Applied Mathematics Letters, 19 (2006), 398-402.

[21] J. Ankudinova, M. Ehrhardt, On the numerical solution of nonlinear Black-Scholes equations, Comput. Math. Appl. 56, (2008), 799-812.

[22] H. Aminikhah, F. Mehrdoust, On approximateanalytical solution of generalized BlackScholes Equation, U.P.B. Sci. Bull., Series A, 77 (4), (2015), 186-194.

[23] G. Hariharan, An Efficient Wavelet Based Approximation Method to Time Fractional Black-Scholes European Option Pricing Problem Arising in Financial Market, Applied 
Mathematical Sciences, 7 (69), (2013), 34453456.

[24] J.H. He, Homotopy perturbation method: A new nonlinear analytical technique, Appl. Math. Comput. 135, (2003), 73-79.

[25] A.S.V. Ravi Kanth, K. Aruna, Comparison of two dimensional DTM and PTDM for solving time-dependent Emden-Fowler Type Equations, International Journal of Nonlinear Science, 13 (2), (2012), 228-239.

[26] A. Ghorbani and J. S. Nadjfi, He's homotopy perturbation method for calculating Adomian's polynomials, Int. J. Nonlin. Sci. Num. Simul. 8 (2) (2007), 229-332.

[27] A. Ghorbani, Beyond Adomian's polynomials: He polynomials, Chaos, Solitons \& Fractals, (2007), 39 (3), (2009), 1486-1492.

[28] L.I.U. Xiaopei, X.U. Genqi, Integral-Type Feedback Controller and Application to the Stabilization of Heat Equation with Boundary Input Delay, WSEAS Transactions on Mathematics, 17, (2018), 311-318.

[29] S.O. Salawu, Analysis of Third-Grade Heat Absorption Hydromagnetic Exothermic Chemical Reactive Flow in a DarcyForchheimer Porous Medium with Convective Cooling, WSEAS Transactions on Mathematics, 17, (2018), 280-289.

[30] S.O. Edeki, G.O. Akinlabi, Zhou Method for the Solutions of System of Proportional Delay Differential Equations, MATEC Web of Conferences, 125, 02001, (2017).

[31] D. Lesnic, The decomposition method for initial value problems, Applied Mathematics and Computation, 181 (2006), 206-213.

[32] W.A. Robin, Solving differential equations using modified Picard iteration, International Journal of Mathematical Education in Science and Technology, 41 (5), (2010), 649-665.

[33] S.O. Edeki, M.E. Adeosun, and E.A. Owoloko, G.O. Akinlabi, I. Adinya, Parameter Estimation of Local Volatility in Currency Option Valuation, International Review on Modelling and Simulations, (2016), 9 (2), 130-133.

[34] K. Abbaoui, Y. Cherruault, Convergence of Adomian's method applied to nonlinear equations, Mathematical and Computer Modelling, 20 (9) (1994) 60-73.

[35] S.O. Edeki, G.O. Akinlabi, Coupled Method for Solving Time-Fractional Navier-Stokes Equation, International Journal of Circuits, Systems and Signal Processing, (2018), 12, 2734.

[36] G.O Akinlabi, S.O Edeki, The Solution of Initial-value Wave-like Models via
Perturbation Iteration Transform Method,

Proceedings of the International Multi Conference of Engineers and Computer Scientists, (2017).

[37] S.O. Edeki, O.O. Ugbebor, E.A. Owoloko, Analytical Solutions of the Black-Scholes Pricing Model for European Option Valuation via a Projected Differential Transformation Method, Entropy, 17 (11), (2015), 7510-7521.

[38] S.O. Edeki, O.O. Ugbebor, and E.A. Owoloko, He's Polynomials for Analytical Solutions of the Black-Scholes Pricing Model for Stock Option Valuation, Proceedings of the World Congress on Engineering, (2016).

[39] M. A. M. Ghandehari and M. Ranjbar, Using Homo-Separation of Variables for Pricing European Option of the Fractional BlackScholes Model in Financial Markets, Mathematical Sciences Letters. 5 (2), (2016), 181-187.

[40] M. Ouafoudi, and F. Gao. Exact Solution of Fractional Black-Scholes European Option Pricing Equations. Applied Mathematics, 9, (2018): 86-100. doi: 10.4236/am.2018.91006.

[41] S. O. Edeki, G. O. Akinlabi and S. A. Adeosun, Analytic and Numerical Solutions of TimeFractional Linear Schrödinger Equation, Communications in Mathematics and Applications, 7 (1), (2016), 1-10.

[42] S. O. Edeki, G. O. Akinlabi, F. O. Egara, A. C. Nzeadibe, Time-fractional classical BlackScholes option pricing model via He-separation of variable transformation method for exact solutions, MMCTSE2020, (2020). 